\title{
Assessing the Relationship between Helicobacter pylori and Chronic Kidney Disease
}

\author{
Koichi Hata ${ }^{1, \dagger}$, Teruhide Koyama ${ }^{1, *,+} \mathbb{C}$, Etsuko Ozaki ${ }^{1}$, Nagato Kuriyama ${ }^{1}$, Shigeto Mizuno ${ }^{2}$, Daisuke Matsui ${ }^{1}$, \\ Isao Watanabe ${ }^{1}$, Ritei Uehara ${ }^{1}$ and Yoshiyuki Watanabe ${ }^{1}$ \\ 1 Department of Epidemiology for Community Health and Medicine, Kyoto Prefectural University of \\ Medicine, 465 Kajii-cho, Kamigyo-ku, Kyoto 602-8566, Japan; s.caballero1229@gmail.com (K.H.); \\ ozaki@koto.kpu-m.ac.jp (E.O.); nkuriyam@koto.kpu-m.ac.jp (N.K.); d-matsui@koto.kpu-m.ac.jp (D.M.); \\ ricky@koto.kpu-m.ac.jp (I.W.); ruehara@koto.kpu-m.ac.jp (R.U.); watanabe@koto.kpu-m.ac.jp (Y.W.) \\ 2 Endoscopy Department, Kindai University Nara Hospital, 1248-1 Otoda-cho, Ikoma, Nara 630-0293, Japan; \\ s-mizuno@med.kindai.ac.jp \\ * Correspondence: tkoyama@koto.kpu-m.ac.jp; Tel.: +81-75-251-5789; Fax: +81-75-251-5799 \\ + These authors contributed equally to this work.
}

Citation: Hata, K.; Koyama, T.; Ozaki, E.; Kuriyama, N.; Mizuno, S.; Matsui, D.; Watanabe, I.; Uehara, R.; Watanabe, Y. Assessing the Relationship between Helicobacter pylori and Chronic Kidney Disease. Healthcare 2021, 9, 162. https://doi. org/10.3390/healthcare 9020162

Academic Editor: Jang-Hee Cho Received: 21 December 2020

Accepted: 31 January 2021

Published: 3 February 2021

Publisher's Note: MDPI stays neutral with regard to jurisdictional claims in published maps and institutional affiliations.

Copyright: (c) 2021 by the authors. Licensee MDPI, Basel, Switzerland. This article is an open access article distributed under the terms and conditions of the Creative Commons Attribution (CC BY) license (https:// creativecommons.org/licenses/by/ $4.0 /)$.

\begin{abstract}
The relationship between Helicobacter pylori infection and/or gastric disorders and chronic kidney disease (CKD) has not been elucidated. We investigated the relationship between Helicobacter pylori and/or atrophic gastritis (AG) and chronic kidney disease. In total, 3560 participants (1127 men and 2433 women) were eligible for this cross-sectional study. We divided participants into four study groups: with/without Helicobacter pylori infection and with/without AG. The HP (+) AG (-) group demonstrated a significant association with CKD compared with the HP (-) AG (-) group (adjusted odds ratio, 1.443; 95\% confidence interval, 1.047-1.989). In contrast, the HP (+) AG (+) group showed significantly lower adjusted odds of CKD than the HP (-) AG (-) group (adjusted odds ratio, 0.608; 95\% confidence interval, 0.402-0.920). H. pylori infection without AG might be associated with CKD in these participants. Conversely, the HP (+) AG (+) group had lower odds of CKD. Uncovering an association between gastric and renal conditions could lead to development of new treatment strategies.
\end{abstract}

Keywords: atrophic gastritis; chronic kidney disease; Helicobacter pylori; inter-organ interactions

\section{Introduction}

The stomach is reported to be associated with conditions affecting other organs. Such as, an association between atrophic gastritis (AG) and coronary artery disease has been described [1,2], with AG representing a potential independent risk factor for coronary artery disease [3]. Some studies have reported that Helicobacter pylori (H. pylori) infection is associated with the metabolic syndrome and abnormal lipid profiles [4,5], while other reports suggest a potential association between $H$. pylori infection and insulin resistance [6,7], liver disorders [8] and ventilator-associated pneumonia [9]. In addition, we have previously suggested that H. pylori infection and AG are useful for risk assessment of osteoporosis [10]. These studies suggested that H. pylori infection and/or AG affect not only the stomach but also other organs.

A previous study reported that individuals infected with $H$. pylori had a higher risk of subsequent renal dysfunction than those not infected [11]. Conversely, the H. pylori infection rate is lower in patients with peptic ulcer disease and concomitant chronic kidney disease (CKD) than in those without CKD [12]. However, the relationship between H. pylori infection and/or gastric disorders and CKD has not been elucidated. Therefore, in this study, we investigated the relationship between H. pylori and/or AG and CKD, in order to determine whether an association exists between the stomach and kidneys. 


\section{Materials and Methods}

\subsection{Study Population}

This cross-sectional study included 4337 individuals enrolled in the Japan MultiInstitutional Collaborative Cohort Study in the Kyoto area, from 2011-2013. The Japan Multi-Institutional Collaborative Cohort Study is a new cohort study, launched in 2005, to examine gene-environmental interactions in lifestyle-related diseases [13]. We excluded participants who had received H. pylori eradication therapy $(n=530)$ or who had undergone gastrectomy $(n=30)$. Further, participants who used a proton-pump inhibitor $(n=112)$, or who had missing medical information data $(n=105)$, were excluded. This left 3560 participants (1127 men and 2433 women) eligible for analysis. Figure 1 shows flow chart of the study participants. The study protocol was approved by the ethics board of Kyoto Prefectural University of Medicine (ethical approval number, RBMR-E-289), and written informed consent was obtained from all participants.

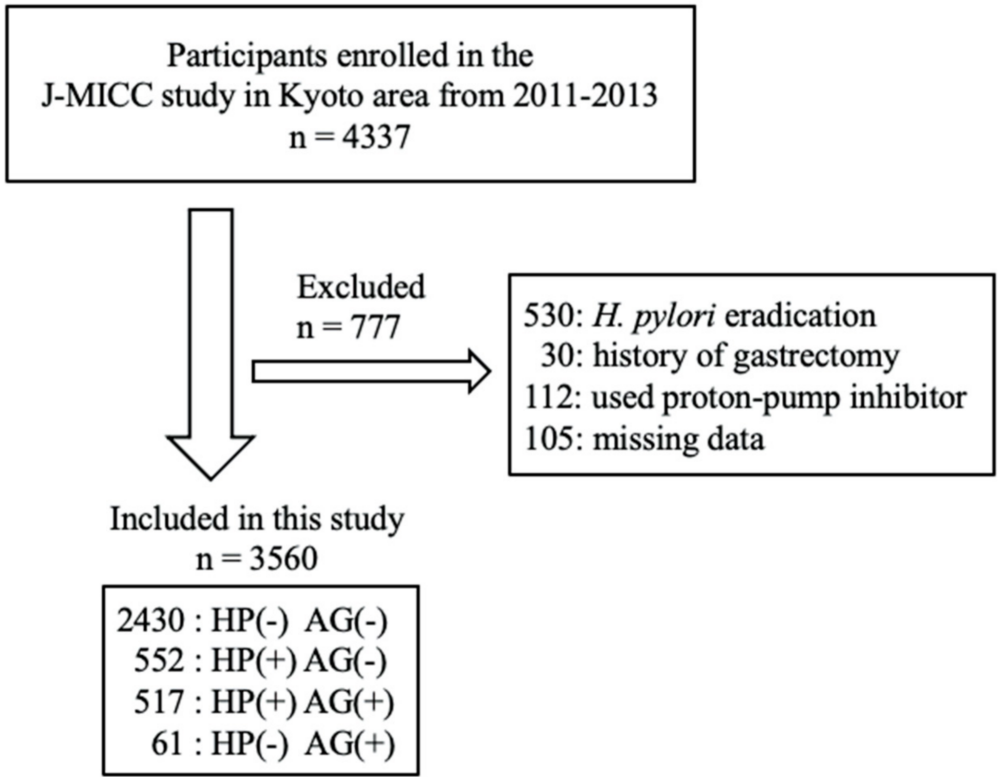

Figure 1. Flow chart of the study participants.

The study evaluated medical information obtained via self-administered questionnaires. Metabolic equivalents (METs) were assessed, as previously reported [14]. Furthermore, blood chemistry data - triglycerides, total cholesterol, high-density lipoprotein (HDL) cholesterol, low-density lipoprotein (LDL) cholesterol, glucose, haemoglobin A1c $(\mathrm{HbA} 1 \mathrm{c})$, uric acid, blood urea nitrogen, creatinine, and pepsinogen (PG) I/II-along with early morning spot urine samples, collected on the day of survey, were assessed. The estimated glomerular filtration rate (eGFR) was calculated using the following equation (JAP-Creatinine): eGFR $\left(\mathrm{mL} / \mathrm{min} / 1.73 \mathrm{~m}^{2}\right)=194 \times$ creatinine $^{-1.094} \times$ year $^{-0.287}$ (for men) and eGFR $\left(\mathrm{mL} / \mathrm{min} / 1.73 \mathrm{~m}^{2}\right)=194 \times$ creatinine $^{-1.094} \times$ year $^{-0.287} \times 0.739$ (for women) [15]. The prevalence of CKD was determined for CKD stages 3-5 (defined as eGFR $<60 \mathrm{~mL} / \mathrm{min} / 1.73 \mathrm{~m}^{2}$ ). Anthropometry data was obtained during the clinical examination. Medical history and medication use were assessed by means of a questionnaire. Hypertension was defined as a resting systolic blood pressure $\geq 140 \mathrm{mmHg}$ or if receiving medication, diabetes mellitus (DM) as $\mathrm{HbA} 1 \mathrm{c} \geq 6.5 \%$ or if receiving medication, dyslipidemia as LDL-cholesterol $\geq 140 \mathrm{mg} / \mathrm{dL}$ and HDL-cholesterol $<40 \mathrm{mg} / \mathrm{dL}$ or if receiving medication, and anaemia as haemoglobin $\leq 13.0 \mathrm{~g} / \mathrm{dL}$ in men and $\leq 12.0 \mathrm{~g} / \mathrm{dL}$ in women.

Participants were divided into four study groups according to a combination of serum anti-H. pylori antibody (HP) positivity and levels of serum PG. This method has recently been used in Japan for gastric cancer screening of high-risk individuals [16-18]. 
In brief, blood samples were obtained, and the serum was separated to measure antiH. pylori antibodies (HP) and PG levels. AG was defined according to the serum PG I and II criteria proposed: when a participant fulfilled the criteria of both serum PG I value $\leq 70 \mathrm{ng} / \mathrm{mL}$ and PG I/II ratio $\leq 3.0$, he or she was diagnosed as having AG. Infection with $H$. pylori was diagnosed using a microplate enzyme immunoassay kit (E Plate Eiken H. pylori Antibody, Eiken Chemical, Tokyo, Japan) [19]. Serum samples were analysed according to the manufacturer's instructions. Participants with a measured value $>10 \mathrm{U} / \mathrm{mL}$ were considered to be infected with $H$. pylori, i.e., HP (+). The control group consisted of participants who tested HP (-) AG (-); while the other three groups consisted of participants who tested HP (+) AG (-), HP (+) AG (+), or HP (-) AG (+).

\subsection{Statistical Analysis}

Analyses were performed using SPSS statistical software (PASW 25.0). For all analyses, $p$ values $<0.05$ were considered statistically significant. Continuous variables were expressed as means \pm standard deviations (SD), and categorical data were expressed as sums and percentages. Inter-group comparisons were performed using the one-way analysis of variance for continuous variables, or the chi-squared test for categorical variables. Categorical variables included sex, alcohol use, smoking, hypertension, DM, dyslipidemia, stroke, myocardial infarction and/or stenocardia, and anaemia. Odds ratios (ORs) and 95\% confidence intervals (CI) were calculated using logistic regression methods in which CKD was the dependent variable and year, sex, body mass index (BMI), METs, smoking, alcohol use, hypertension, DM, dyslipidemia, stroke, myocardial infarction and/or stenocardia, and anaemia were the independent variables.3.

\section{Results}

Table 1 shows the characteristics of participants according to $H$. pylori infection and AG. The mean age of the control group was 50.0 years, vs. $>54.0$ years for the other groups. Table 2 shows the distribution of HP, AG, and CKD within age strata. In each group, the prevalence of CKD increased as year increased. However, CKD prevalence in the HP $(+)$ AG (-) group in the age stratum 50-59 years was approximately double that of the control group.

Table 3 shows the proportion of participants with/without CKD, stratified by H. pylori infection and diagnosis of AG. Relative to the control group, the HP (+) AG (-) group had an adjusted OR for CKD of 1.465 (95\% CI, 1.066-2.012) adjusted for yeae and sex; 1.439 (95\% CI, 1.046-1.979) adjusted for year, sex, BMI, METs, alcohol use, and smoking; and 1.443 (95\% CI, 1.047-1.989) adjusted for year, sex, BMI, METs, smoking, alcohol use, hypertension, stroke, dyslipidemia, myocardial infarction and/or stenocardia. There was no significant difference in the adjusted odds of CKD between the control group and the HP (+) AG (-) group—adjusted for lifestyle factors (BMI, METs, alcohol use, and smoking) or medical history (hypertension, stroke, dyslipidemia, myocardial infarction and / or stenocardia). In contrast, we compared the HP (+) AG (+) group and HP (-) AG $(-)$ group, the HP (+) AG (+) group had lower an adjusted OR for CKD of $0.610(95 \% \mathrm{CI}$, $0.406-0.917)$ adjusted for year and sex. There was no significant difference in the odds of CKD between the control group and the HP (+) AG (+) group, adjusted for lifestyle factors or medical history. On the other hand, the HP (-) AG (+) group showed no significant association with CKD. 
Table 1. Characteristics of subjects according to HP infection and/or AG status.

\begin{tabular}{|c|c|c|c|c|c|c|c|c|c|}
\hline \multirow{3}{*}{ Variable } & \multicolumn{2}{|c|}{ HP (-) AG (-) } & \multicolumn{2}{|c|}{ HP (+) AG (-) } & \multicolumn{2}{|c|}{ HP (+) AG (+) } & \multicolumn{2}{|c|}{$\mathrm{HP}(-) \mathrm{AG}(+)$} & \multirow{3}{*}{$p$-Value } \\
\hline & \multicolumn{2}{|c|}{$n=2430$} & \multicolumn{2}{|c|}{$n=552$} & \multicolumn{2}{|c|}{$n=517$} & \multicolumn{2}{|c|}{$n=61$} & \\
\hline & Mean (n) & SD (\%) & Mean (n) & SD (\%) & Mean (n) & SD (\%) & Mean (n) & SD (\%) & \\
\hline Sex (male) & (744) & $(30.6)$ & $(200)$ & $(36.2)$ & $(160)$ & $(30.9)$ & (23) & $(37.7)$ & 0.053 \\
\hline Age (years) & 50.0 & 10.1 & 54.4 & 9.80 & 57.7 & 8.86 & 57.5 & 9.80 & $<0.001$ \\
\hline $\mathrm{BMI}\left(\mathrm{kg} / \mathrm{m}^{2}\right)$ & 21.9 & 3.06 & 22.4 & 3.24 & 22.1 & 3.08 & 22.2 & 3.64 & 0.004 \\
\hline Systolic blood pressure (mmHg) & 130 & 20.3 & 135 & 20.8 & 137 & 21.6 & 132 & 21.0 & $<0.001$ \\
\hline Diastolic blood pressure (mmHg) & 77.9 & 11.8 & 79.9 & 12.1 & 80.3 & 11.5 & 77.8 & 13.1 & $<0.001$ \\
\hline Total cholesterol (mg/dL) & 213 & 37.2 & 219 & 37.3 & 218 & 36.3 & 216 & 34.2 & 0.002 \\
\hline Triglyceride (mg/dL) & 128 & 102 & 146 & 102 & 129 & 90.8 & 128 & 78.4 & 0.001 \\
\hline HDL-cholesterol (mg/dL) & 72.2 & 19.4 & 68.4 & 18.2 & 69.6 & 18.6 & 71.5 & 20.1 & $<0.001$ \\
\hline LDL-cholesterol (mg/dL) & 120 & 31.6 & 126 & 31.4 & 126 & 31.6 & 122 & 25.7 & $<0.001$ \\
\hline Glucose (mg/dL) & 92.3 & 20.2 & 94.3 & 24.4 & 95.3 & 22.2 & 96.0 & 25.7 & 0.009 \\
\hline Hemoglobin (g/dL) & 13.6 & 1.41 & 13.7 & 1.40 & 13.5 & 1.33 & 13.6 & 1.71 & 0.035 \\
\hline Hemoglobin A1C (\%) & 5.37 & 0.42 & 5.46 & 0.58 & 5.48 & 0.49 & 5.49 & 0.55 & $<0.001$ \\
\hline Uric acid (mg/dL) & 5.19 & 2.29 & 5.30 & 2.32 & 5.25 & 2.50 & 5.59 & 3.03 & 0.456 \\
\hline BUN (mg/dL) & 13.9 & 4.04 & 14.7 & 4.16 & 14.2 & 4.27 & 14.4 & 6.32 & $<0.001$ \\
\hline Creatinine (mg/dL) & 0.69 & 0.15 & 0.72 & 0.18 & 0.67 & 0.14 & 0.84 & 1.31 & $<0.001$ \\
\hline $\mathrm{eGFR}\left(\mathrm{mL} / \mathrm{min} / 1.73 \mathrm{~m}^{2}\right)$ & 79.9 & 13.9 & 76.1 & 14.5 & 79.0 & 13.8 & 78.8 & 17.0 & $<0.001$ \\
\hline PG I $(\mathrm{ng} / \mathrm{mL})$ & 49.1 & 19.8 & 81.9 & 44.6 & 44.0 & 17.2 & 25.9 & 16.6 & $<0.001$ \\
\hline PG II (ng/mL) & 9.28 & 3.75 & 27.3 & 16.1 & 23.1 & 8.60 & 13.5 & 6.87 & $<0.001$ \\
\hline PG I/II ratio & 5.44 & 1.28 & 3.40 & 1.36 & 1.92 & 0.63 & 1.90 & 0.84 & $<0.001$ \\
\hline METs (h/day) & 12.4 & 10.3 & 13.2 & 10.6 & 14.1 & 10.6 & 16.6 & 12.6 & $<0.001$ \\
\hline Current smokers & $(269)$ & $(11.1)$ & $(68)$ & $(12.3)$ & $(58)$ & $(11.2)$ & (10) & $(16.4)$ & 0.522 \\
\hline Current drinkers & (1484) & (61.1) & (324) & $(58.7)$ & (281) & $(54.4)$ & (34) & (55.7) & 0.034 \\
\hline Hypertension & $(794)^{\prime}$ & (32.7) & (232) & $(42.0)$ & $(244)$ & (47.2) & (22) & (36.1) & $<0.001$ \\
\hline Diabetes mellitus & $(58)$ & $(2.4)^{\prime}$ & $(28)$ & $(5.1)$ & $(29)^{\prime}$ & $(5.6)^{\prime}$ & $(4)^{\prime}$ & $(6.6)^{\prime}$ & $<0.001$ \\
\hline Dyslipidemia & (790) & (32.5) & $(242)$ & $(43.8)$ & (229) & $(44.3)$ & (21) & (34.4) & $<0.001$ \\
\hline Anemia & (248) & $(10.2)$ & $(51)$ & $(9.2)$ & (65) & (12.6) & (8) & (13.1) & 0.265 \\
\hline $\begin{array}{l}\text { Myocardial infarction } \\
\text { and/or stenocardia }\end{array}$ & (33) & (1.4) & (11) & $(2.0)$ & (8) & $(1.5)$ & (1) & $(1.6)$ & 0.738 \\
\hline Stroke & (22) & $(0.9)$ & (4) & $(0.7)$ & (6) & $(1.2)$ & (1) & (1.6) & 0.825 \\
\hline
\end{tabular}

HP, Helicobactor pylori; AG, atrophic gastritis; BMI, body mass index; HDL, high density lipoprotein; LDL, low density lipoprotein; BUN, blood urea nitrogen; GFR, glomerular filtration rate; PG, pepsinogen; METs, metabolic equivalents. $p$-values were determined using one-way ANOVA or chi-squared test between status.

Table 2. The distribution of HP infection and/or AG status and CKD according to year-grade.

\begin{tabular}{|c|c|c|c|c|c|c|c|c|}
\hline \multirow{3}{*}{ Year } & \multicolumn{2}{|c|}{$\mathrm{HP}(-)$ AG (-) } & \multicolumn{2}{|c|}{$\mathrm{HP}(+) \mathrm{AG}(-)$} & \multicolumn{2}{|c|}{ HP (+) AG (+) } & \multicolumn{2}{|c|}{$\mathrm{HP}(-) \mathrm{AG}(+)$} \\
\hline & \multicolumn{2}{|c|}{ CKD } & \multicolumn{2}{|c|}{ CKD } & \multicolumn{2}{|c|}{ CKD } & \multicolumn{2}{|c|}{ CKD } \\
\hline & $(-)$ & $(+)$ & $(-)$ & $(+)$ & $(-)$ & $(+)$ & $(-)$ & $(+)$ \\
\hline \multirow{2}{*}{$35-49$} & 1262 & 23 & 179 & 5 & 97 & 2 & 13 & 0 \\
\hline & $98.2 \%$ & $1.8 \%$ & $97.3 \%$ & $2.7 \%$ & $98.0 \%$ & $2.0 \%$ & $100.0 \%$ & $0.0 \%$ \\
\hline \multirow[b]{2}{*}{$50-59$} & 516 & 44 & 134 & 21 & 125 & 12 & 15 & 2 \\
\hline & $92.1 \%$ & $7.9 \%$ & $86.5 \%$ & $13.5 \%$ & $91.2 \%$ & $8.8 \%$ & $88.2 \%$ & $11.8 \%$ \\
\hline \multirow[b]{2}{*}{$60-69$} & 502 & 83 & 173 & 40 & 263 & 18 & 26 & 5 \\
\hline & $85.8 \%$ & $14.2 \%$ & $81.2 \%$ & $18.8 \%$ & $93.6 \%$ & $6.4 \%$ & $83.9 \%$ & $16.1 \%$ \\
\hline
\end{tabular}

Table 3. Multivariate adjusted associations of CKD with HP infection and/or AG.

\begin{tabular}{|c|c|c|c|c|c|c|c|c|c|c|c|}
\hline & \multicolumn{2}{|c|}{ CKD } & \multirow{2}{*}{$\mathrm{OR}^{+}$} & \multirow{2}{*}{$95 \% \mathrm{CI}^{\dagger}$} & \multirow{2}{*}{$p$-Value } & \multirow{2}{*}{$\mathrm{OR}^{++}$} & \multirow{2}{*}{$95 \% \mathrm{CI}^{++}$} & \multirow{2}{*}{$p$-Value } & \multirow{2}{*}{ OR $\S$} & \multirow{2}{*}{$95 \% \mathrm{CI}^{\S}$} & \multirow{2}{*}{$p$-Value } \\
\hline & $(-)$ & $(+)$ & & & & & & & & & \\
\hline $\mathrm{HP}(-) \mathrm{AG}(-)$ & 2280 & 150 & Reference & & & Reference & & & Reference & & \\
\hline $\mathrm{HP}(+) \mathrm{AG}(-)$ & 486 & 66 & 1.465 & $1.066-2.012$ & 0.018 & 1.439 & $1.046-1.979$ & 0.025 & 1.443 & $1.047-1.989$ & 0.025 \\
\hline $\mathrm{HP}(+) \mathrm{AG}(+)$ & 485 & 32 & 0.610 & $0.406-0.917$ & 0.017 & 0.615 & $0.408-0.926$ & 0.020 & 0.608 & $0.402-0.920$ & 0.019 \\
\hline $\mathrm{HP}(-) \mathrm{AG}(+)$ & 54 & 7 & 1.126 & $0.492-2.573$ & 0.779 & 1.134 & $0.492-2.614$ & 0.768 & 1.076 & $0.456-2.539$ & 0.867 \\
\hline
\end{tabular}

CKD, chronic kidney disease; HP, Helicobactor pylori; AG, atrophic gastritis; OR, odds ratio; CI, confidence interval. + Adjusted for year, sex. †† Adjusted for year, sex, BMI, METs, drinking and smoking. § Adjusted for year, sex, BMI, METs, drinking and smoking, hypertension, DM, dyslipidemia, stroke, myocardial infarction and/or stenocardia, and anemia.

\section{Discussion}

Associations between two or multiple organs are essential for the human body to maintain homeostasis and to function normally. Elucidating these associations may be important for clinical decision-making and the development of appropriate treatments 
for some diseases. With knowledge of interactions between organ systems, we can treat diseases focusing not only on the symptomatic organ, but also on the organ fundamentally causing the disease. Moreover, such knowledge should allow for prediction and prevention of other related-organ disorders when a patient suffers from a particular organ disorder. Indeed, $H$. pylori infection not only affects the stomach, but is associated with other risk factors for CKD: hypertension, metabolic syndrome, DM, cardiovascular disease, changes in lipid profile [11]. In this study, we focused on the relationship between the kidneys and the stomach, in terms of $H$. pylori infection and/or AG.

Previous meta-analyses have reported that association of $H$. pylori infection and CKD and revealed no association between $H$. pylori infection and either non-dialysis [20] or dialysis-dependent patients [21]. On the other hand, a meta-analysis evaluated the prevalence of $H$. pylori infection in patients with CKD and concluded that there is a lower prevalence of $H$. pylori infection in patients with CKD [22]. The reported risk of CKD in patients with $H$. pylori infection is still conflicting. Given that $H$. pylori inhabits the stomach, it is necessary to consider the state of AG when demonstrating the relationship between H. pylori infection and CKD. Although it has been that the risk factor for peptic ulcer are $H$. pylori infection [23], the rate of $H$. pylori infection is lower in individuals with peptic ulcer disease and concomitant CKD than in those without CKD [12]. Thus, it has been suggested that gastric disorders, such as peptic ulcer disease, may influence the relationship between CKD and $H$. pylori infection. Indeed, in the present study, the HP (+) AG (-) and HP (+) AG (+) groups demonstrated an inverse association with CKD. If we examine only the presence or absence of $H$. pylori infection and the risk of CKD without considering the presence or absence of AG, the results are inconsistent. Furthermore, in the 50-59-year-old age stratum, the $\mathrm{HP}(+) \mathrm{AG}(-)$ group had a CKD prevalence approximately double that of the control group. CKD prevalence and $H$. pylori infection are strongly associated with age $[24,25]$. This study also showed CKD prevalence was greater among 60-69-year-olds than other age stratum. The effect of the H. pylori infection without AG may have been accelerated the onset of CKD in the 50-59-year-old age stratum.

These results suggest that the prevalence of $\mathrm{HP}(+) \mathrm{AG}(-)$ status is higher in CKD and may have relationship with the risk of CKD, an effect that has been speculated by one mechanism: that $H$. pylori infection reduces ghrelin secretion. Ghrelin, a gastrointestinal peptide hormone, is known to perform a plethora of central and peripheral actions in distinct areas, including gut motility, gastric acid secretion, and glucose metabolism [26] There are two different circulating forms of this peptide: acylated ghrelin, the active form that binds to receptors, and des-acylated ghrelin, the inactive form without affinity for receptors $[27,28]$. The level of acylated ghrelin is more important than total ghrelin in terms of clarifying the effect of ghrelin. Ghrelin can improve CKD via complex interactions affecting energy homeostasis, appetite, muscle mitochondrial activity, suppression of inflammation, and maintenance of the cardiovascular system $[29,30]$. H. pylori infection is associated with decreased ghrelin production and with a reduction in the number of ghrelinproducing cells [31,32]. In contrast, higher levels of acylated ghrelin have been observed in patients with chronic AG compared with healthy participants [33]. This suggests that there may be a compensatory increase in plasma acylated ghrelin concentration in response to $A G$, a condition that results in a loss of ghrelin-producing cells and an increase in gastric $\mathrm{pH}$ [32]. Therefore, in this study, we speculate that the HP (+) AG (-) and HP (+) AG $(+)$ were observed association with CKD may be due to decreased or increased levels of acylated ghrelin caused by H. pylori infection with/without AG. Furthermore, there was no difference in the relationship between CKD and lifestyle factors and medical history between the control and HP (+) AG (-) group. In sum, H. pylori infection may induce renal dysfunction via reduction of ghrelin, and further studies should be conducted to confirm this hypothesis (Figure 2 showed the hypothesis in this study.). 


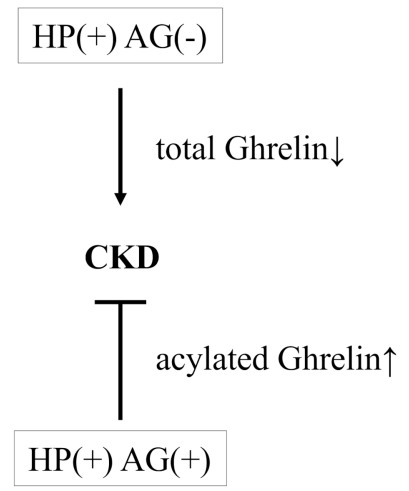

Figure 2. The hypothesis in this study.

The limitations of the present study are mainly related to the study design and lack of measured ghrelin and proteinuria. The present study was cross-sectional; thus, we could not investigate the incidence of CKD in relation to $H$. pylori infection. A prospective study is warranted to assess the relationships between CKD and H. pylori. Although the study evaluated medical information obtained via self-administered questionnaires, some anti-diabetes drugs used for other reasons or for pre-diabetes, like metformin and we can't detect diabetics from non-diabetics. Furthermore, we could not show direct evidence of an interaction between ghrelin levels and H. pylori infection and/or AG status. In addition, CKD is generally defined in terms of proteinuria and eGFR; however, we had limited data on proteinuria. Last, we diagnosed $H$. pylori infection via serum antibody detection, whereas the gold standard is gastric-based testing.

\section{Conclusions}

We demonstrated that the prevalence of HP (+) AG (-) status is higher in CKD and may have relationship with the risk of CKD. H. pylori, an important pathogenic factor in the stomach, is probably involved in the development of many other diseases. Uncovering the association between gastric and renal conditions could lead to the development of new treatment strategies. In theory, if a patient suffers from $H$. pylori infection, we could predict renal disorders and potentially prevent their development by treating the stomach, for example, by administering $H$. pylori eradication treatment.

Author Contributions: Conceptualization, K.H. and T.K.; methodology, T.K.; formal analysis, K.H. and T.K.; investigation and data curation, E.O., N.K., D.M., I.W.; writing-original draft preparation, K.H. and T.K.; writing-review and editing, T.K.; visualization, K.H. and T.K.; supervision, S.M. and R.U.; project administration, Y.W.; funding acquisition, Y.W. All authors have read and agreed to the published version of the manuscript.

Funding: This study was supported in part by a Grant-in-Aid for Scientific Research on Priority Areas of Cancer (No. 17015018), Grant-in-Aid for Scientific Research on Innovative Areas (No. 221S0001), and JSPS KAKENHI Grant Number 16H06277, 25460233.

Institutional Review Board Statement: The study protocol was approved by the ethics board of Kyoto Prefectural University of Medicine (ethical approval number, RBMR-E-289).

Informed Consent Statement: Informed consent was obtained from all subjects involved in the study.

Data Availability Statement: The data presented in this study are available on request from the corresponding author.

Conflicts of Interest: The authors declare that they have no conflict of interest.

\section{References}

1. Takashima, T.; Adachi, K.; Kawamura, A.; Yuki, M.; Fujishiro, H.; Rumi, M.A.; Ishihara, S.; Watanabe, M.; Kinoshita, Y. Cardiovascular risk factors in subjects with Helicobacter pylori infection. Helicobacter 2002, 7, 86-90. [CrossRef] [PubMed] 
2. Vijayvergiya, R.; Vadivelu, R. Role of Helicobacter pylori infection in pathogenesis of atherosclerosis. World J. Cardiol. 2015, 7, 134-143. [CrossRef] [PubMed]

3. Senmaru, T.; Fukui, M.; Tanaka, M.; Kuroda, M.; Yamazaki, M.; Oda, Y.; Naito, Y.; Hasegawa, G.; Toda, H.; Yoshikawa, T.; et al. Atrophic gastritis is associated with coronary artery disease. J. Clin. Biochem. Nutr. 2012, 51, 39-41. [CrossRef] [PubMed]

4. Gunji, T.; Matsuhashi, N.; Sato, H.; Fujibayashi, K.; Okumura, M.; Sasabe, N.; Urabe, A. Helicobacter pylori infection is significantly associated with metabolic syndrome in the Japanese population. Am. J. Gastroenterol. 2008, 103, 3005-3010. [CrossRef] [PubMed]

5. Satoh, H.; Saijo, Y.; Yoshioka, E.; Tsutsui, H. Helicobacter Pylori infection is a significant risk for modified lipid profile in Japanese male subjects. J. Atheroscler. Thromb. 2010, 17, 1041-1048. [CrossRef] [PubMed]

6. Imai, J.; Yamada, T.; Saito, T.; Ishigaki, Y.; Hinokio, Y.; Kotake, H.; Oka, Y.; Katagiri, H. Eradication of insulin resistance. Lancet 2009, 374, 264. [CrossRef]

7. Polyzos, S.A.; Kountouras, J.; Zavos, C.; Deretzi, G. The association between Helicobacter pylori infection and insulin resistance: A systematic review. Helicobacter 2011, 16, 79-88. [CrossRef]

8. Waluga, M.; Kukla, M.; Zorniak, M.; Bacik, A.; Kotulski, R. From the stomach to other organs: Helicobacter pylori and the liver. World J. Hepatol. 2015, 7, 2136-2146. [CrossRef]

9. Dadashi, A.; Hosseinzadeh, N. High seroprevalence of anti-Helicobacter pylori antibodies in patients with ventilator-associated pneumonia. J. Res. Med. Sci. 2018, 23, 79. [CrossRef]

10. Mizuno, S.; Matsui, D.; Watanabe, I.; Ozaki, E.; Kuriyama, N.; Watanabe, Y. Serologically Determined Gastric Mucosal Condition Is a Predictive Factor for Osteoporosis in Japanese Men. Dig. Dis. Sci. 2015, 60, 2063-2069. [CrossRef]

11. Lin, S.Y.; Lin, C.L.; Liu, J.H.; Yang, Y.F.; Huang, C.C.; Kao, C.H. Association between Helicobacter pylori infection and the subsequent risk of end-stage renal disease: A nationwide population-based cohort study. Int. J. Clin. Pract. 2015, 69, 604-610. [CrossRef] [PubMed]

12. Chang, S.S.; Hu, H.Y. Lower Helicobacter pylori infection rate in chronic kidney disease and end-stage renal disease patients with peptic ulcer disease. J. Chin. Med Assoc. JCMA 2014, 77, 354-359. [CrossRef] [PubMed]

13. Wakai, K.; Hamajima, N.; Okada, R.; Naito, M.; Morita, E.; Hishida, A.; Kawai, S.; Nishio, K.; Yin, G.; Asai, Y.; et al. Profile of Participants and Genotype Distributions of 108 Polymorphisms in a Cross-Sectional Study of Associations of Genotypes With Lifestyle and Clinical Factors: A Project in the Japan Multi-Institutional Collaborative Cohort (J-MICC) Study. J. Epidemiol. 2011, 21, 223-235. [CrossRef] [PubMed]

14. Koyama, T.; Matsui, D.; Kuriyama, N.; Ozaki, E.; Tanaka, K.; Oze, I.; Hamajima, N.; Wakai, K.; Okada, R.; Arisawa, K.; et al. Genetic variants of SLC17A1 are associated with cholesterol homeostasis and hyperhomocysteinaemia in Japanese men. Sci. Rep. 2015, 5, 15888. [CrossRef] [PubMed]

15. Matsuo, S.; Imai, E.; Horio, M.; Yasuda, Y.; Tomita, K.; Nitta, K.; Yamagata, K.; Tomino, Y.; Yokoyama, H.; Hishida, A. Revised equations for estimated GFR from serum creatinine in Japan. Am. J. Kidney Dis. Off. J. Natl. Kidney Found. 2009, 53, 982-992. [CrossRef]

16. Miki, K. Gastric cancer screening by combined assay for serum anti-Helicobacter pylori IgG antibody and serum pepsinogen levels-“ABC method". Proc. Jpn. Acad. Ser. B 2011, 87, 405-414. [CrossRef]

17. Ohata, H.; Kitauchi, S.; Yoshimura, N.; Mugitani, K.; Iwane, M.; Nakamura, H.; Yoshikawa, A.; Yanaoka, K.; Arii, K.; Tamai, H.; et al. Progression of chronic atrophic gastritis associated with Helicobacter pylori infection increases risk of gastric cancer. Int. J. Cancer 2004, 109, 138-143. [CrossRef]

18. Watabe, H.; Mitsushima, T.; Yamaji, Y.; Okamoto, M.; Wada, R.; Kokubo, T.; Doi, H.; Yoshida, H.; Kawabe, T.; Omata, M. Predicting the development of gastric cancer from combining Helicobacter pylori antibodies and serum pepsinogen status: A prospective endoscopic cohort study. Gut 2005, 54, 764-768. [CrossRef]

19. Kawai, T.; Kawakami, K.; Kudo, T.; Ogiahara, S.; Handa, Y.; Moriyasu, F. A new serum antibody test kit (E plate) for evaluation of Helicobacter pylori eradication. Intern. Med. 2002, 41, 780-783. [CrossRef]

20. Wijarnpreecha, K.; Thongprayoon, C.; Nissaisorakarn, P.; Jaruvongvanich, V.; Nakkala, K.; Rajapakse, R.; Cheungpasitporn, W. Association of Helicobacter pylori with Chronic Kidney Diseases: A Meta-Analysis. Dig. Dis. Sci. 2017, 62, 2045-2052. [CrossRef]

21. Gu, M.; Xiao, S.; Pan, X.; Zhang, G. Helicobacter pylori Infection in Dialysis Patients: A Meta-Analysis. Gastroenterol. Res. Pract. 2013, 2013, 785892. [CrossRef] [PubMed]

22. Shin, S.P.; Bang, C.S.; Lee, J.J.; Baik, G.H. Helicobacter pylori Infection in Patients with Chronic Kidney Disease: A Systematic Review and Meta-Analysis. Gut Liver 2019, 13, 628-641. [CrossRef] [PubMed]

23. Sayehmiri, K.; Abangah, G.; Kalvandi, G.; Tavan, H.; Aazami, S. Prevalence of peptic ulcer in Iran: Systematic review and meta-analysis methods. J. Res. Med. Sci. 2018, 23, 8. [CrossRef] [PubMed]

24. Glassock, R.J.S.; Rule, A.D. Aging and the Kidneys: Anatomy, Physiology and Consequences for Defining Chronic Kidney Disease. Nephron 2016, 134, 25-29. [CrossRef] [PubMed]

25. Inoue, M. Changing epidemiology of Helicobacter pylori in Japan. Gastric Cancer 2017, 20, 3-7. [CrossRef] [PubMed]

26. Muller, T.D.; Nogueiras, R.; Andermann, M.L.; Andrews, Z.B.; Anker, S.D.; Argente, J.; Batterham, R.L.; Benoit, S.C.; Bowers, C.Y.; Broglio, F.; et al. Ghrelin. Mol. Metab. 2015, 4, 437-460. [CrossRef] [PubMed]

27. Hosoda, H.; Kojima, M.; Matsuo, H.; Kangawa, K. Ghrelin and des-acyl ghrelin: Two major forms of rat ghrelin peptide in gastrointestinal tissue. Biochem. Biophys. Res. Commun. 2000, 279, 909-913. [CrossRef] 
28. Hosoda, H.; Kojima, M.; Matsuo, H.; Kangawa, K. Purification and characterization of rat des-Gln14-Ghrelin, a second endogenous ligand for the growth hormone secretagogue receptor. J. Biol. Chem. 2000, 275, 21995-22000. [CrossRef]

29. Cheung, W.W.; Mak, R.H. Ghrelin in chronic kidney disease. Int. J. Pept. 2010, 2010. [CrossRef]

30. Gunta, S.S.; Mak, R.H. Ghrelin and leptin pathophysiology in chronic kidney disease. Pediatr. Nephrol. 2013, $28,611-616$. [CrossRef]

31. Paoluzi, O.A.; Blanco del, V.G.; Caruso, R.; Monteleone, I.; Monteleone, G.; Pallone, F. Impairment of ghrelin synthesis in Helicobacter pylori-colonized stomach: New clues for the pathogenesis of H. pylori-related gastric inflammation. World J. Gastroenterol. 2014, 20, 639-646. [CrossRef] [PubMed]

32. Isomoto, H.; Ueno, H.; Saenko, V.A.; Mondal, M.S.; Nishi, Y.; Kawano, N.; Ohnita, K.; Mizuta, Y.; Ohtsuru, A.; Yamashita, S.; et al. Impact of Helicobacter pylori infection on gastric and plasma ghrelin dynamics in humans. Am. J. Gastroenterol. 2005, 100, 1711-1720. [CrossRef] [PubMed]

33. Campana, D.; Nori, F.; Pagotto, U.; De Iasio, R.; Morselli-Labate, A.M.; Pasquali, R.; Corinaldesi, R.; Tomassetti, P. Plasma acylated ghrelin levels are higher in patients with chronic atrophic gastritis. Clin. Endocrinol. 2007, 67, 761-766. [CrossRef] [PubMed] 